\title{
PERSPECTIVE IN NANOSCIENCE
}

\section{The New Era of Nutraceuticals: Beneficial Effects of Polyphenols in Various Experimental and Clinical Settings}

\author{
Thea Magrone* and Emilio Jirillo \\ Department of Basic Medical Sciences, Neuroscience and Sensory Organs, University of Bari, School of Medicine, Bari, Italy
}

Worldwide there is a growing interest on nutrition and, in particular, on Mediterranean diet which is mainly based on assumption of fruit, vegetables, cereals and extravirgin olive oil. Polyphenols are compounds largely distributed in the vegetal kingdom, exerting beneficial effects on human health due to their anti-oxidant, anti-inflammatory and anti-allergic properties [1]. Polyphenols are subdivided into two main categories: flavonoids and non flavonoids (e.g., resveratrol). Flavonoids consist of two aromatic rings, benzypiran carbon rings ( $\mathrm{A}$ and $\mathrm{C}$ rings) and benzene (B rings) and may be divided into six subclasses according to degree of the oxidation of the C-ring, the hydroxylation pattern of the ring structure and the substitutions at the 3position [1]. Resveratrol is structurally characterized by the presence of a 1,2-diphenylethylene nucleus with hydroxyls substituted in the aromatic rings [1].

Polyphenols act on the immune and non-immune cells, thanks to different receptors such as aryl hydrocarbon receptor (AHR) located on the cytoplasm and combined with heat shock protein 90 and the co-chaperone 23 [2]. After activation, AHR is able to interact with polyphenols, thus, triggering several responses. For example, at intestinal level AHR is present on intraepithelial lymphocytes, T (h) helper 17 cells, innate lymphoid cells, dendritic cells and macrophages, thus participating to main immune responses.

From a pathological point of view, in inflammatory bowel disease, such as Crohn disease (CD) and ulcerative colitis (UC), it has been shown that cellular distribution of AHR is reduced in CD patients in comparison to UC patients even if there were no differences in AHR gene expression [2]. Therefore, this condition may be due to modifications occurring at posttranscriptional levels. In murine experimental sodium dextrane sulphate-induced colitis, it has been observed that AHR activation down-regulated release of pro-inflammatory cytokines, while inducing production of interleukin (IL)-22 [3]. Of note, these events mediate an anti-inflammatory effect. In in vitro murine experimental colitis and in vivo in human UC subjects, the beneficial effects exerted by polyphenols have been ascribed to their ability to inhibit NF- $\mathrm{KB}$ pathway and tumor necrosis factor- $\alpha$ release [4]. Finally, other authors highlighted the role of gut microbiota on the production of polyphenol metabolites endowed with anti-inflammatory activities [5].

The effects exerted by polyphenols have been experimented in cardiovascular disease (CVD) which is a major cause of morbidity and mortality. It is well known that moderate intake of red wine (French paradox) has represented the first evidence about the preventative effect of polyphenol assumption in relation to CVD outcome [6]. In fact, French people from the Bordeaux region, who usually assume moderate amounts of red wine, despite a diet rich in saturated fatty acids exhibit lower risk of CVD mortality in comparison to American people with the same hypercaloric diet but in the absence of red wine intake [6]. In this direction, it has also been shown that polyphenols can exert anti-fibrotic and myocardial protection via inhibition of oxidative stress and molecular pathways that are involved in heart fibrosis [7]. Moderate red wine assumption may also lead to vasodilation through nitric oxide (NO) release by both endothelial cells and monocytes, ultimately, culminating to amelioration of vascular function [8]. In fact, NO release increases cyclic guanosine monophosphate (cGMP), thus, leading to smooth muscle relaxation and inhibition of platelet aggregation, on the one hand [9]. On the other hand, cGMP increases production of prostacyclin, which, in turn, decreases plasma leukotrienes provoking vasodilation [9]. Furthermore, also consumers of cocoa and dark chocolate containing polyphenols benefit of this effect and, in particular, flow mediated dilation was increased in comparison with lower polyphenol consumers [10]. Other authors have conducted a metabolomic study utilizing Sakura green tea leaves, dark chocolate and turmeric powder, demonstrating a superior beneficial effects of polyphenols, also avoiding high calorie intake [11]. This combination seems to be even more appropriate for preventing CVD outcome. Also an in vitro study using peripheral blood mononuclear cells (PBMCs) treated with polyphenols extracted from olive leaves documented an increase in NO production by monocytes and a potential protective effect on CVD outcome [12]. In extravirgin olive oil (EVOO), hydroxytyrosol, the main polyphenol present in free form, acts as a scavenger of reactive oxygen species (ROS), also exerting beneficial cardiovascular effects through decrease in oxidized low density lipoprotein and platelet aggregation [13]. Therefore, it has been proposed that consumption of EVOO may contribute to the anti-inflammatory and cardioprotective effects of the Mediterranean diet, thus, preventing atherosclerosis and CVD [14].

It is well known that type II diabetes leads to high risk of CVD outcome. Quite interestingly, a few studies have demonstrated the protective effects of polyphenols in diabetes outcome. In fact, human clinical trials documented the antidiabetic effects by resveratrol overcoming insulin resistance by reducing blood glucose and glycated hemoglobin A1c levels [15].

*Address correspondence to this author at the Aggregate Professor of Immunology, Department of Basic Medical Sciences, Neuroscience and Sensory Organs, University of Bari, School of Medicine, Bari, Italy; P.zza Giulio Cesare 11, 70124 Bari, Bari, Italy; Tel: +39 0805478492; E-mail: thea.magrone@uniba.it 
Another important issue is the capacity of polyphenols to modulate liver function, an organ which acts as a filter in order to detoxify external and internal noxious agents. In liver, grape polyphenol extracts are able to inhibit ROS, decrease proinflammatory cytokine levels, enhance hypoxia tolerance and modulate lipid metabolism [16]. Furthermore, Louvet and Mathurin [17] described the beneficial effects of polyphenols from Litsea coreana via inhibition of hepatic adipose differentiation-related proteins. In vitro treatment of hepatocytes from non-alcoholic fatty liver disease patients with polyphenols gave rise to a reduction of lipogenesis and an increase in acid $\beta$-oxidation [18]. Gut metabolites of blackberry fruits increased glucose consumption and glycogen levels in hepatic cells, restoring glutathione and mitochondrial membrane potential [19]. Polyphenols extracted from green tea possess anti-viral hepatic activity, and, in particular, in an in vitro study epigallocatechin-3-gallate was able to interfere with entry of hepatitis B virus via inhibition of clathrin-mediated endocytosis [20]. Furthermore, in hepatitis $\mathrm{C}$ virus (HCV) disease, delphidin from green tea could interfere with envelope proteins of $\mathrm{HCV}$, causing a deformation of virus particles and, thus, impairing their interaction with cell plasma membrane [21].

Obesity represents another serious problem that involves a great number of subjects both in childhood and adulthood as a result of an incorrect life style such as type of diet and reduced physical activity. In a recent report, evidence has been provided that supplementation of polyphenols from red grape to human obese PBMCs led to an anti-inflammatory pathway with increase in IL-10 secretion and reduction of IL-21 [22]. In a clinical trial, obese females, who ingested polyphenols extracted from Ibiscus sabdariffa and Lipia citriodora, underwent weight reduction with normalization of blood pressure, on the one hand [23]. On the other hand, same authors have also pointed out the modulation of satiety exerted by polyphenols in virtue of a normalization of leptin release and reduction of resistin. Furthermore, administration of blackberry to overweight and obese males increased fat oxidation and improved insulin sensitivity [24].

Over the past decades, allergic diseases have been increasing, thus, representing an emergency in medicine. Many studies based on the administration of polyphenols have been conducted to solve allergic manifestations. For example, since ultraviolet A (UVA) radiation induces damage at dermal level, human skin dermal fibroblasts were irradiated with UVA source in the presence of polyphenols [25]. In this model, polyphenols prevented damage-associated UVA exposure through reduction of ROS release, pro-inflammatory markers and improvement of mitochondrial function via anti-oxidant enzyme expression. In peanut allergic disease, polyphenol extracts inhibited IgE binding to rat basophilic leukemia cells, thus, suppressing their degranulation [26]. Human peripheral blood basophils activated with IgE were in vitro treated with fermented grape marc (FGM). Results demonstrated that FGM polyphenols were able to inhibit basophil degranulation and increase release of IL-10 by $\mathrm{T}$ regulatory cells, dampening IL-4 production by Th2 cells and release of IgE [27]. Furthermore, an in vitro study using human PBMCs from nickel-sensitized patients in the presence of red grape polyphenols clearly demonstrated a reduction of oxidative stress and of cytokines release such as interferon- $\gamma$ and IL-17, with an increase in IL-10 production [28]. Conclusively, polyphenols were able to promote a tolerogenic pathway in nickel-mediated allergic contact dermatitis.

Finally, polyphenols in virtue of their properties are able to exert a neuroprotective role and may be used to attenuate the symptoms of Parkinson's disease (PD) and Alzheimer disease (AD) [29]. Particularly, the ability of polyphenols to act on aggregation and disaggregation of amyloid peptide, tau, $\alpha$-synuclein and synphilin-1, have been pointed out.

Furthermore, it has in vitro been demonstrated, using human striatal precursor cells, that addition of polyphenols led to an increase in cell proliferation and pancadherin, which is a protein important for synaptic plasticity, and focal adhesion kinase during development of the central nervous system [30].

Conclusively, polyphenols express a broad spectrum of beneficial effects on different cell types and organs either in health or in disease. Despite their described effects, future studies should be aimed at identifying the major metabolites of polyphenols following their intestinal absorption. This should be a very important step in the preparation of compounds which directly target specific cells also defining optimal concentrations to attain healthy effects.

\section{CONFLICT OF INTEREST}

The authors declare no conflict of interest, financial or otherwise.

\section{ACKNOWLEDGEMENTS}

This paper was supported by "Intervento cofinanziato dal Fondo di Sviluppo e Coesione 2007-2013-APQ Ricerca Regione Puglia Programma regionale a sostegno della specializzazione intelligente e della sostenibilità sociale ed ambientaleFutureInResearch".

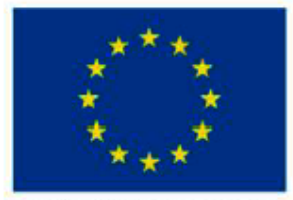

UNIONE EUROPEA
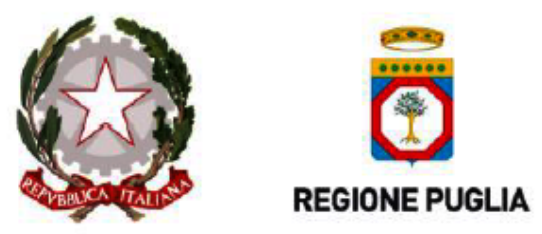

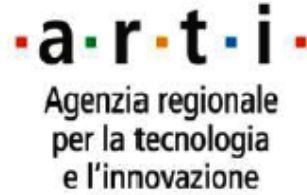




\section{REFERENCES}

[1] Magrone T, Jirillo E. Effects of Polyphenols on Inflammatory-Allergic Conditions: Experimental and Clinical Evidences. In: Watson R, Preedy V. Polyphenols: Prevention and Treatment of Human Disease. Eds. Elsevier 2018, Second Edition, Vol. 2 pp. 253-59.

[2] Lamas B, Natividad JM, Sokol H. Aryl hydrocarbon receptor and intestinal immunity. Mucosal Immunol 2018; 11(4): 1024-38.

[3] Monteleone I, Rizzo A, Sarra M, et al. Aryl hydrocarbon receptor-induced signals up-regulate IL-22 production and inhibit inflammation in the gastrointestinal tract. Gastroenterology 2011; 141(1): 237-48, 48.e1.

[4] Shapira S, Leshno A, Katz D, et al. Of mice and men: a novel dietary supplement for the treatment of ulcerative colitis. Therap Adv Gastroenterol 2017; 11: $1756283 X 17741864$

[5] Dudek-Wicher RK, Junka A, Bartoszewicz M. The influence of antibiotics and dietary components on gut microbiota. Prz Gastroenterol 2018; 13(2): 85-92.

[6] Snopek L, Mlcek J, Sochorova L, et al. Contribution of Red Wine Consumption to Human Health Protection. Molecules 2018; $23(7)$. pii: E1684.

[7] Zhang N, Wei WY, Li LL, Hu C, Tang QZ. Therapeutic Potential of Polyphenols in Cardiac Fibrosis. Front Pharmacol 2018 ; 9 : 122.

[8] Oak MH, Auger C, Belcastro E, Park SH, Lee HH, Schini-Kerth VB. Potential mechanisms underlying cardiovascular protection by polyphenols: Role of the endothelium. Free Radic Biol Med 2018; 122: 161-70.

[9] Giglio RV, Patti AM, Cicero AFG, et al. Polyphenols: Potential Use in the Prevention and Treatment of Cardiovascular Diseases. Curr Pharm Des 2018; 24(2): 239-58.

[10] Magrone T, Russo MA, Jirillo E. Cocoa and Dark Chocolate Polyphenols: From Biology to Clinical Applications. Front Immunol 2017 ; 8: 677.

[11] Martini S, Conte A, Tagliazucchi D. Comprehensive evaluation of phenolic profile in dark chocolate and dark chocolate enriched with Sakura green tea leaves or turmeric powder. Food Res Int 2018; 112: 1-16.

[12] Magrone T, Spagnoletta A, Salvatore R, et al. Olive leaf extracts act as modulators of the human immune response. Endocr Metab Immune Disord Drug Targets 2018; 18(1): 85-93.

[13] Casas R, Estruch R, Sacanella E. The Protective Effects of Extra Virgin Olive Oil on Immune-mediated Inflammatory Responses. Endocr Metab Immune Disord Drug Targets 2018; 18(1): 23-35.

[14] Wongwarawipat T, Papageorgiou N, Bertsias D, Siasos G, Tousoulis D. Olive Oil-related Anti-inflammatory Effects on Atherosclerosis: Potential Clinical Implications Endocr Metab Immune Disord Drug Targets 2018; 18(1): 51-62.

[15] Cao H, Ou J, Chen L, et al. Dietary polyphenols and type 2 diabetes: Human study and clinical trials. Crit Rev Food Sci Nutr 2011; 1-19. doi: 10.1080/10408398.2018.1492900.

[16] Olivero-David R, Ruiz-Roso MB, Caporaso N, et al. In vivo bioavailability of polyphenols from grape by-product extracts, and effect on lipemia of normocholesterolemic Wistar rats. J Sci Food Agric 2018; 98(115): 5581-90.

[17] LouvetA, Mathurin P. Alcoholic liver disease: mechanisms of injury and targeted treatment. Nat Rev Gastroenterol Hepatol 2015; $12(4): 231-42$.

[18] Rodriguez-Ramiro I, Vauzour D, Minihane AM. Polyphenols and non-alcoholic fatty liver disease: impact and mechanisms. Proc Nutr Soc 2016; 75(1): 47-60.

[19] Gowd V, Bao T, Wang L, et al. Antioxidant and antidiabetic activity of blackberry after gastrointestinal digestion and human gut microbiota fermentation. Food Chem 2018; 269: 618-27.

[20] Wu YH. Naturally derived anti-hepatitis B virus agents and their mechanism of action. World J Gastroenterol 2016; 22(1): 188-204.

[21] Calland N, Sahuc ME, Belouzard S, et al. Polyphenols Inhibit Hepatitis C Virus Entry by a New Mechanism of Action. J Virol 2015; 89(19): 1005363 .

[22] Magrone T, Jirillo E, Spagnoletta A, et al. Immune Profile of Obese People and In Vitro Effects of Red Grape Polyphenols on Peripheral Blood Mononuclear Cells. Oxid Med Cell Longev 2017, 2017: 9210862.

[23] Boix-Castejón M, Herranz-López M, Pérez Gago A, et al. Hibiscus and lemon verbena polyphenols modulate appetite-related biomarkers in overweight subjects: a randomized controlled trial. Food Funct 2018; 9(6): 3173-84.

[24] Solverson PM, Rumpler WV, Leger JL, et al. Blackberry Feeding Increases Fat Oxidation and Improves Insulin Sensitivity in Overweight and Obese Males. Nutrients 2018; 10(8): pii: E1048.

[25] Gasparrini M, Forbes-Hernandez TY, Afrin S, et al. Strawberry-Based Cosmetic Formulations Protect Human Dermal Fibroblasts against UVAInduced Damage. Nutrients 2017; 9(6): pii: E605.

[26] Bansode RR, Plundrich NJ, Randolph PD, Lila MA, Williams LL. Peanut flour aggregation with polyphenolic extracts derived from peanut skin inhibits IgE binding capacity and attenuates RBL-2H3 cells degranulation via MAPK signaling pathway. Food Chem 2018; $263: 307-14$.

[27] Marzulli G, Magrone T, Vonghia L, et al. Immunomodulating and anti-allergic effects of Negroamaro and Koshu Vitis vinifera fermented grape marc (FGM). Curr Pharm Des 2014; 20(6): 864-8.

[28] Magrone T, Romita P, Verni P, et al. In vitro effects of polyphenols on the peripheral immune responses in nickel-sensitized patients. Endocr Metab Immune Disord Drug Targets 2017; 17: 324-31.

[29] Freyssin A, Page G, Fauconneau B, Rioux Bilan A. Natural polyphenols effects on protein aggregates in Alzheimer's and Parkinson's prion-like diseases. Neural Regen Res 2018; 13(6): 955-61.

[30] Sarchielli E, Morelli A, Guarnieri G, Iorizzi M, Sgambati E. Neuroprotective effects of quercetin 4'-O- $\beta$-d-diglucoside on human striatal precursor cells in nutrient deprivation condition. Acta Histochem 2018; 120(2): 122-8. 